\title{
BIOECONOMÍA Y MODELOS PRODUCTIVOS SOSTENIBLES EN ORGANIZACIONES DE AGRICULTORES: UNA REVISIÓN (PÓSTER)
}

\author{
Natalia Lajara-Camilleri $^{a *}$, Alicia Mateos-Ronco $^{a}$ \\ ${ }^{a}$ Centro de Investigación en Gestión de Empresas (CEGEA), Universitat Politècnica de València, Camino \\ de Vera, s/n, 46022 Valencia-nalade@upv.es; amateos@cegea.upv.es
}

La aprobación de los Objetivos de Desarrollo Sostenible (ODS) en 2015, en el marco de la Agenda 2030 de Naciones Unidas, ha marcado un punto de inflexión, a nivel académico y en las políticas de investigación y transferencia, en relación a la presión medioambiental y la necesidad de desarrollar economías sostenibles. Uno de los retos expresados por la Unión Europea en relación con la bioeconomía se centra en la agricultura sostenible. La misión fundamental de las cooperativas agroalimentarias actualmente es aglutinar la oferta y ejercer de vehículo transmisor de las demandas del mercado. Son un agente que puede tener un rol indiscutible en la transición hacia modelos productivos más sostenibles. Este trabajo presenta una revisión de la literatura para identificar experiencias de transformación agro-sostenible en organizaciones de agricultores y el papel que estas organizaciones tienen en la transición hacia una economía sostenible. Los resultados evidencian la inexistencia de estándares o normas para la evaluación de la sostenibilidad agraria en las explotaciones. Además, el papel de las cooperativas como entidades impulsoras de estrategias agro-sostenibles es evidente, al incentivar la adopción de dichas estrategias por los productores agrarios, si bien se detectan diferencias en los resultados relativos al tipo de prácticas fomentadas.

Palabras clave: bioeconomía, economía circular, sector agroalimentario, cooperativas, sostenibilidad.

\section{Introducción y objetivos}

En 2015, la Asamblea General de Naciones Unidas aprobaba la resolución conocida como Agenda 2030. Se trata de un paso adelante en el compromiso con la trasformación hacia un modelo sostenible, un «plan de acción en favor de las personas, el planeta y la prosperidad». En base a 17 objetivos seleccionados como ejes de acción (ODS) se desarrollan las 169 metas establecidas en relación al desarrollo sostenible en el ámbito económico, social y ambiental. En este escenario han ido surgiendo distintas iniciativas encaminadas a ayudar en el cumplimiento de los compromisos adquiridos. La Comisión Europea presentó en marzo de 2020 un Plan de acción para la Economía Circular, que busca principalmente atajar el problema desde la raíz: regulando el diseño de productos sostenibles, la reducción de residuos o el empoderamiento del ciudadano vía información y derechos (a reparar).

El sector agroalimentario, sin ser destinatario específico de este plan, se ve afectado directamente en la necesidad de reducir residuos y avanzar en la transición desde la economía lineal hacia un modelo circular. El Pacto Verde Europeo, presentado por la Comisión Europea en diciembre de 2019, contempla en su estrategia «De la granja a la mesa (From farm to fork)» la necesidad de contar con sistemas de producción más eficientes, un mejor almacenamiento y envasado, llevar a cabo una transformación y transporte agrícola más sostenibles, posibilitar un consumo saludable al tiempo que se reduzca la pérdida y desperdicio de alimentos y formar ciudadanos mejor informados. En definitiva, se trata de lograr un equilibrio entre la naturaleza, los sistemas alimentarios y la biodiversidad para proteger la salud de los ciudadanos, incrementando al mismo tiempo la competitividad y resiliencia de la Unión Europea.

El compromiso con estas medidas se extiende más allá de los gobiernos firmantes y permea a todos los niveles de sus sociedades, tanto a nivel público como privado. El reto es colosal y no ha hecho sino verse acrecentado con la situación pandémica causada por el COVID-19. La propia Agenda 2030 reconoce que el sector privado es clave para conseguir las metas fijadas y subraya explícitamente el rol de las cooperativas en esta tarea. Efectivamente, en el sector agroalimentario, específicamente en el contexto de la producción y comercialización en España, las cooperativas desempeñan un papel indiscutible como vehículos de transmisión de las demandas del mercado hacia los productores que, con frecuencia, son pequeños y con escasa capacidad de negociación [Lajara-Camilleri y Server, 2017]. Además, los productores suelen depositar las decisiones empresariales en cuanto a estrategia e innovación en manos de la cooperativa con la que comercializan su producción. Ello convierte a este tipo de organización en un agente imprescindible en la transición hacia modelos más sostenibles.

No obstante, hasta el momento son escasos los trabajos científicos que han analizado el grado de integración de los ODS en la gestión empresarial [Van der Waal et al., 2021]. La medición de la interacción de las empresas con los ODS es una cuestión complicada debido a las múltiples interrelaciones que existen entre los propios ODS [Schaltegger, 2018]. Según Pizzi et al. (2020), estas limitaciones son la causa fundamental de que los estudios previos se hayan abordado desde una perspectiva basada en evidencias, para detectar las fortalezas y debilidades que influyen en el comportamiento de las empresas. El objetivo de este trabajo 
es realizar una revisión sistemática de los trabajos publicados en la literatura científica para identificar prácticas sostenibles que, en el contexto de una economía circular, se están desarrollando en cooperativas agrarias. En concreto, se pretende identificar (i) las principales áreas temáticas en torno a las cuales se están desarrollando más experiencias y (ii) los factores de motivación que determinan que las cooperativas se decidan por la adopción de este tipo de procesos.

\section{Metodología}

Se realiza una revisión sistemática [Cook et al., 1997]. Esta metodología se caracteriza por una definición precisa del propósito del trabajo, una búsqueda integral con selección relevante de información mediante criterios explícitos, una valoración crítica de los resultados obtenidos y decisiones reproducibles respecto a relevancia, selección y rigor metodológico. El enfoque adoptado para la revisión es el de síntesis narrativa, según metodología propuesta por Denyer y Tranfield (2008). La ventaja es que da cabida a trabajos y estudios diversos, el proceso de revisión es flexible y permite explorar temas novedosos, así como identificar buenas prácticas.

La búsqueda se ha llevado a cabo en mayo de 2021 utilizando las bases de datos de trabajos científicos Scopus y Web of Science, identificando trabajos que combinaran, bien en el título o como palabras clave, los términos «Agro-food cooperative», «Agri-food cooperative» con «sustainability», «circular economy», «agro-sustainability», «life cycle assessment»o «food waste». Los únicos resultados considerados fueron los artículos científicos en inglés o español. No se introdujeron límites temporales. Los resultados obtenidos en Web of Science fueron 18 trabajos y en Scopus se localizaron 37 artículos.

\section{Resultados}

El creciente grado de interés que despierta la sostenibilidad y el modelo de economía circular en el mundo cooperativo agroalimentario queda reflejado en la evolución del número de artículos científicos publicados en los últimos años, especialmente desde 2017 [Lampridi et al., 2019].

El papel de las cooperativas en la transición hacia una agricultura sostenible radica fundamentalmente en ejercer de entidades impulsoras de estrategias agro-sostenibles que incentiven la adopción de las mismas por parte de los productores agrarios, aspecto que se manifiesta de forma recurrente en la literatura científica. Yang et al. (2016), en relación con la adopción de estrategias sostenibles de cultivo de hortalizas en invernadero, identifican la necesidad de adoptar un sistema de gestión integrada de nutrientes y la selección de especies vegetales de baja acumulación de metales, con objeto de reducir los riesgos medioambientales. Para ello es necesaria una gestión unificada de inputs agrícolas y una elevada eficiencia de los servicios de extensión agrícola. Las cooperativas, como organizaciones de pequeños productores, se constituyen en este contexto como el vehículo que debe impulsar la adopción de estas estrategias sostenibles, con objeto de estabilizar los rendimientos agrícolas y, en consecuencia, incrementar los beneficios de los productores, lo que a su vez estimulará la iniciativa de los agricultores por la adopción de este tipo de prácticas.

Este efecto positivo entre sostenibilidad y el rol de las cooperativas queda patente también en el trabajo de Candemir et al. (2021), en el que se realiza una completa revisión de los artículos publicados que vinculan a las cooperativas agroalimentarias con los tres pilares de la sostenibilidad: económica, social y medioambiental. En él hace hincapié en el valor añadido que aportan este tipo de entidades especialmente en países en vías de desarrollo, donde la mejora en la productividad y renta de los socios es más notable. El trabajo cita las investigaciones de González (2018) a la hora de afirmar que, en conjunto, las cooperativas agroalimentarias influyen positivamente en los agricultores a la hora de adoptar prácticas medioambientales e innovación, incrementando, por tanto, la sostenibilidad medioambiental de las explotaciones. En esta misma línea Bro et al. (2019) identifican los factores determinantes de la adopción de prácticas de producción sostenible en productores de café y determinan hasta qué punto la pertenencia a la cooperativa impacta en la adopción de dichas prácticas. Sus resultados revelan una relación positiva entre ambos aspectos, así como un incremento en la probabilidad de adopción mayor en los productores cooperativistas que en el resto. Sin embargo, sí evidencian diferencias significativas en relación al tipo de prácticas sostenibles adoptadas. La pertenencia a la cooperativa estimula la adopción de estrategias que promueven la conservación de los recursos hídricos pero, a diferencia de otras investigaciones [Yang et al., 2016], no es relevante para las prácticas que promueven la salud del suelo y los cultivos, ni para las relativas al manejo de la tierra.

Entre las variables que influyen en las decisiones de los productores agrarios en relación a la adopción de prácticas sostenibles, Yu et al. (2021) identifican la aversión al riesgo. En su trabajo analizan el impacto combinado de la aversión al riesgo de los productores de hortalizas y su pertenencia a una cooperativa en la adopción de técnicas "verdes" de producción, así como en qué medida la pertenencia a la cooperativa inhibe esta aversión natural. Sus resultados evidencian que la aversión al riesgo tiene un impacto significativo y positivo sobre su pertenencia a la cooperativa, pero significativo negativo en la adopción de prácticas "verdes" de gestión agrícola. En este contexto, el papel de la cooperativa se configura no sólo 
como facilitador de la utilización de prácticas agro-sostenibles sino como mitigador del efecto inhibidor de la aversión al riesgo sobre dicha adopción.

Silva et al. (2021) señalan un aspecto esencial que en ocasiones pasa desapercibido en las primeras etapas de la transición de modelos, donde la atención suele centrarse en el seno de las organizaciones y deja fuera del foco las relaciones inter-organizacionales. Los autores analizan cómo la sostenibilidad puede transmitirse a lo largo de la cadena de valor. Para ello analizan las cadenas de suministro de la miel y el anacardo en Brasil, utilizando un enfoque cualitativo a través de entrevistas y comparando la actividad de empresas mercantiles con el funcionamiento de las cooperativas de agricultores. Los resultados obtenidos ponen de manifiesto que las señas identitarias del cooperativismo, especialmente la toma de decisiones democrática y la difusión de la información, ayudan a generar mecanismos directos de gobernanza que redundan en una mayor diseminación de los requerimientos de sostenibilidad. En esta misma línea, el estudio empírico desarrollado por Pérez-Mesa et al. (2021) sobre una muestra de cooperativas de Almería concluye que las relaciones entre la distribución y las cooperativas de comercialización, que antes se concretaban en términos de calidad, han sido superadas y reemplazadas por el concepto de sostenibilidad, tanto en el ámbito económico, como social y medioambiental. Los resultados señalan también la necesidad, por una parte, de llevar a cabo integraciones horizontales o verticales (preferentemente en el sentido de sus proveedores) y, por otra, de adoptar herramientas y tecnologías que permitan transformar la cadena de suministro agroalimentaria. También Fuss et al. (2021) concluyen que la modificación de ciertos procesos de reciclado de residuos hacia prácticas propias de la economía circular debe realizarse en consonancia con los procesos de transformación de la industria primaria. Para ello es preciso potenciar la comunicación eficaz y el entendimiento mutuo a lo largo de la cadena de los sistemas de reciclaje socio-integrados, minimizando la visión cortoplacista y única del ahorro de costes a favor de tendencias que incidan en un desarrollo de la economía circular sostenible y local.

En relación a estudios sectoriales, las cooperativas españolas de aceite de oliva han sido analizadas utilizando análisis cualitativo comparativo [Rabadán et al., 2021], con objeto de evaluar a los impulsores de la eco-innovación. Los resultados señalan que las grandes cooperativas de este sector tienen un elevado compromiso con la sostenibilidad y que la colaboración, especialmente con un número considerable de agentes, estimula la eco-innovación, de forma particularmente notable en empresas pequeñas. En el sector del vino existen diversos trabajos publicados, como el de Luzzani et al. (2021), en el que se presenta una herramienta que apoya la gestión integrada de la sostenibilidad en bodegas (de tipo cooperativo o no). Este recurso aporta información sobre debilidades y fortalezas de las estrategias de gestión relacionadas con la sostenibilidad con el propósito de ayudar en la toma de decisiones en la empresa. El artículo recoge resultados correspondientes a 47 empresas del sector vinícola italiano, siendo 13 de ellas, cooperativas.

\section{Conclusiones}

La economía circular es un objetivo clave en el Pacto Verde Europeo. La Comisión Europea establece la economía circular como una de las prioridades de investigación e innovación para un futuro sostenible. Este hecho queda patente en el interés mostrado por la comunidad científica en el tema, como revela el número creciente de trabajos publicados en los últimos años.

Los resultados de la revisión sistemática realizada ofrecen varias aportaciones. Por una parte, la investigación de las distintas metodologías utilizadas para la evaluación de la sostenibilidad agraria en las explotaciones es especialmente relevante ya que no existen, actualmente, estándares o normas para dicha evaluación [Lampridi et al., 2019]. Los métodos más utilizados incluyen herramientas, marcos e índices basados en indicadores, seguidos de las metodologías multicriterio, si bien hay que destacar que es frecuente la utilización simultánea de varias metodologías combinadas.

El papel de las cooperativas como agentes impulsores de prácticas sostenibles por los productores del sector agroalimentario a nivel mundial queda claramente evidenciado, si bien hay que destacar disparidades en los resultados relativos al tipo de prácticas fomentadas. Este hecho, no obstante, puede atribuirse a los condicionantes de los diferentes cultivos, zonas y sistemas de producción e incluso localización geográfica de los diferentes trabajos empíricos.

Con objeto de conseguir los retos de la economía circular, asegurando la perdurabilidad de materiales y productos, es preciso implementar y escalar modelos de negocio circular que además aseguren la participación de los distintos stakeholders, así como ahondar en el estudio de criterios eficientes de asignación de recursos, legitimación y valores culturales esenciales para dotar de operatividad a las prácticas de economía circular.

También se enfatiza el papel de las alianzas y relaciones inter-organizacionales [Geissdoerfer et al., 2020] a lo largo de la cadena de valor como facilitador de las diferentes estrategias. En este sentido, la sustantividad y señas de identidad propias de las cooperativas ayudan a crear los mecanismos de cooperación que facilitan alcanzar los requerimientos de sostenibilidad. Por todo ello, es preciso potenciar el papel de las cooperativas como medio para promover la adopción de prácticas sostenibles, reduciendo el 
riesgo individual asumido por los productores y mejorando las condiciones internas y externas que faciliten la transición hacia una economía circular.

\section{Bibliografía}

Bro, A.S.; Clay, D.C.; Ortega, D.L.; López, M.C. (2019). "Determinants of adoption of sustainable production practices among smallholder coffee producers in Nicaragua". Environmental Development Sustainable, 21:895-915.

Candemir, A.; Duvaleix, S.; Latruffe, L. (2021). "Agricultural cooperatives and farm sustainability - a literature review". Journal of Economic Surveys. DOI: 10.1111/joes.12417

Cook, D.J.; Mulrow, C.D.; Haynes, B. (1997). "Systematic Reviews: Synthesis of Best Evidence for Clinical Decisions". Annals of Internal Medicine, 126.

Denyer, D.; Tranfield, D. (2008). "Using qualitative research synthesis to build an actionable knowledge base". Management Decision, 44:213-227.

Esposito, B.; Sessa, M.R.; Sica, D.; Malandrino, O. (2020). "Towards circular economy in the agri-food sector. A systematic literature review". Sustainability, 12:7401.

Fuss, M.; Barros, R.T.V.; Poganietz, W.R. (2021). "The role of a socio-integrated recycling system in implementing a circular economy. The case of Belo Horizonte, Brazil". Waste Management, 121: 215-225.

Geissdoerfer, M.; Pieroni, M.P.P.; Pigosso, D.C.A.; Soufani, K. (2020). "Circular business models: a review". Journal of Cleaner Production, 227:123741.

González, R.A: (2018). Farmers' cooperatives and Sustainable Food Systems in Europe. Routledge.

Lajara-Camilleri, N.; Server Izquierdo, R. (2017): “¿Cómo se puede mejorar la competitividad de las cooperativas agroalimentarias?". CIRIEC-España, Revista de Economía Pública, Social y Cooperativa, 90:103-121.

Lampridi, M.G.; Sorensen, C.G.; Bochtis, D. (2019). "Agricultural sustainability: a review of concepts and methods". Sustainability, 11: 5120.

Luzzani, G; Lamastra, L.; Valentino, F.; Capri, E. (2021). "Development and implementation of a qualitative framework for the sustainable management of wine companies". Science of the Total Environment, 759.

Pérez-Mesa, J.C.; Piedra-Muñoz, L.; Galdeano-Gómez, E.; Giagnocavo, C. (2021). "Management Strategies and Collaborative Relationships for Sustainability in the Agrifood Supply Chain". Sustainability, 13:749.

Pizzi, S.; Caputo, A.; Corvino, A.; Venturelli, A. (2020). "Management research and the UN sustainable development goals (SDGs): A bibliometric investigation and systemic review". Journal of Cleaner Production, 276.

Rabadan, A.; Alvarez-Orti, M; Tello; J.; Pardo, JE. (2021). "Tradition vs Eco-innovation: the constraining effect of Protected Designation of Origin (PDO) on the implementation of sustainability measures in the olive oil sector". Agronomy, 11(3).

Silva, M.E.; Dias, G.P.; Gold, S. (2021). "Exploring the roles of lead organizations in spreading sustainability standards throughout food supply chains in an emerging economy". International Journal of Logistics Management. DOI:10.1108/IJLM-05-2020-0201

Van der Waal, J.W.H.; Thijssens, T.; Maas, K. (2021). "The innovation contribution of multinational enterprises to the Sustainable Development Goals". Journal of Cleaner Production, 285.

Yang, L.; Huang, B.; Mao, M.; Yao, L.; Niedermann, S.; Hu, W.; Chen, Y. (2016). "Sustainability assessment of greenhouse vegetable farming practices from environmental, economic and socioinstitutional perspectives in China". Environmental Science and Pollution Research, 23: 1728717297.

Yu, L.; Chen, C.; Niu, Z.; Gao, Y.; Yang, H.; Xue, Z. (2021). "Risk aversion, cooperative membership and the adoption of green control techniques: evidence from China". Journal of Cleaner Production, 279:123 288 